\title{
Good Vibrations: Asymmetric Vibrations for Directional Haptic Cues
}

\author{
Hanns W. Tappeiner* \\ Robotics Institute \\ Carnegie Mellon University
}

\author{
Roberta L. Klatzky ${ }^{\dagger}$ \\ Department of Psychology \\ Carnegie Mellon University
}

\author{
Bert Unger \\ Robotics Institute \\ Carnegie Mellon University
}

\author{
Ralph Hollis ${ }^{\S}$ \\ Robotics Institute \\ Carnegie Mellon University
}

\begin{abstract}
In this paper we present a new concept for haptic guidance in multiple dimensions, in the form of asymmetric vibrations. We show that by adding asymmetry, vibrations from a single source can, like continuous forces, provide effective, high-resolution directional haptic cues in multiple dimensions. Unlike feedback via continuous forces, vibrations can be generated in an almost arbitrarily small workspace. After describing the concept in detail, we describe a user study that assesses the effects of such vibrations - applied to subjects' hands - on their ability to distinguish between different directions. The results indicate that asymmetric vibrations can provide accurate directional haptic feedback.
\end{abstract}

\section{INTRODUCTION}

When users of a haptic interface explore or guide objects in an unknown environment, it is highly useful, if not critical, to provide directions for approach or avoidance of obstacles. Cues may be given audibly or visually, but haptic feedback offers the advantages of being directly processed and useful in an environment where only force cues are provided. Resisting forces are typically used to signal impact with channels or walls, but these come too late when impact is to be avoided. Another approach is to use a force field that offers resistance that is monotonically related to the distance from a channel or barrier, but this interferes with the haptic control itself.

We describe a new approach to haptic guidance based on asymmetric vibrations. Generating haptic feedback via vibrations is a widely used technique in research and simple versions have been implemented in a variety of consumer products. Robust onedimensional vibrations using a single transducer, such as a mass on a motor, are used in cell phones. One reason why vibrations are used frequently for haptic feedback is because transducers can be very small and are cheap and easy to produce.

More complex setups using multiple transducers to give users a sense of directionality are still research projects. Numerous studies have been performed exploring the effects of vibratory feedback for alerting or for providing an impression of direction. In [1], Lindeman et al. show how vibrations generated by multiple simple transducers placed on a subject's body affect a real world "building clearing" task by giving haptic hints. Ho, Tan and Spence demonstrated in [2] how vibrations can be used to draw a driver's attention to possible hazards. A similar system is available now in certain luxury cars, where the steering wheel and/or seat starts to vibrate to signal a cars out of lane drift to the driver.

Okamura et al. implemented a system for haptic feedback via vibrations for virtual environments and show that vibrations can

\footnotetext{
*e-mail: hanns@cs.cmu.edu

†e-mail:klatzky@cmu.edu

†e-mail:bgu@andrew.cmu.edu

§e-mail:rhollis@cs.cmu.edu
}

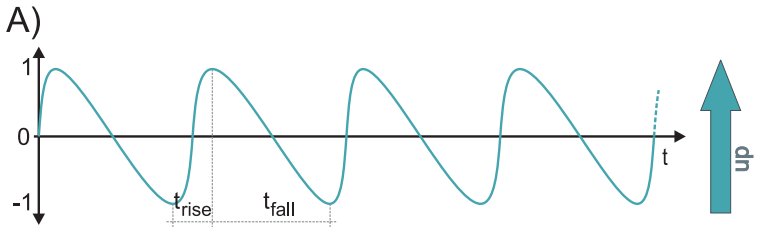

B)
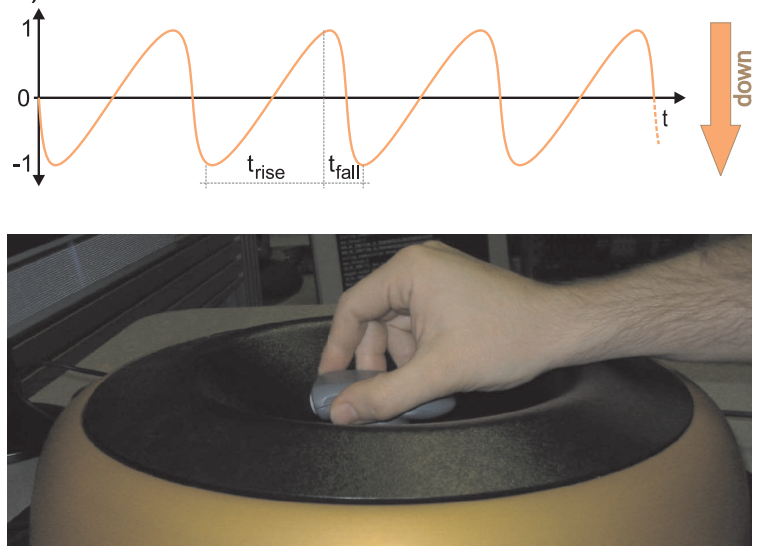

Figure 1: A) shows an asymmetric sinusoid with short rise time and long fall time, so the power of the rising part is higher. B) shows the inverse of $A$ ), with the falling part having higher power. Over time, A) feels like an up motion, B) like a down motion

significantly enhance touch perception for virtual environment applications ([3] and [4]).

Very interesting related work has been done by Amemiya et al. to use one dimensional, asymmetric oscillations generated by a motor/mass system to provide directional cues in a non grounded, hand held device [5] or to manipulate the perceived weight of a hand held object [6]. The results are encouraging and show that, in some cases, an asymmetrically accelerated mass can provide useful directional feedback and change a subjects weight perception in the direction of the higher acceleration.

\subsection{Motivation}

While mechanical motor/mass systems like the one described by Amemiya can provide feedback in one dimension (or potentially two dimesions with additional hardware), the setup becomes unfeasible for higher dimensions. In our work, we focus on multi dimensional workspaces and high resolution directional feedback. We are interested in how well people can distinguish between different directions from cues generated by asymmetric vibrations. Also, the system proposed in this paper is able to change all parameters of the vibrations on the fly, including amplitude. On the other hand, our system is by no means a hand held device which can be carried around, but a full, high fidelity 6DOF desktop haptic device.

Even though continuous forces can be used for directional hap- 


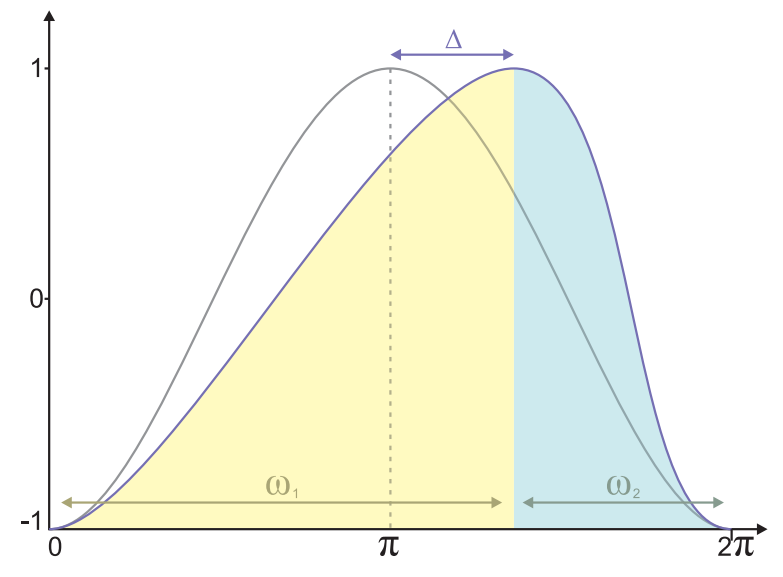

Figure 2: One period of an asymmetric sinusoidal function, composed of two half periods of different length.

tic feedback in a haptic device with a reasonably large workspace as well, we believe that asymmetric vibrations can have certain advantages, especially if they can be superimposed with continuous forces. In this paper we try to determine whether asymmetric vibrations are suitable for generating multi directional haptic cues. In subsequent work, we would like to compare haptic feedback from continuous forces vs. asymmetric vibrations qualitatively and explore possibilities on how they could be superimposed.

For haptics, one advantage of vibrations over continuous forces is that powerful vibrations can be generated with very small amplitudes, using up only a fraction of the haptic device's workspace. A second advantage is that vibrations add one more parameter for possible feedback: time. With continuous forces, stronger feedback is achieved by larger forces. With vibrations, stronger feedback can be generated by greater amplitude, higher frequencies, or both, depending on the current need of the application. The above approaches have in common that they use one-dimensional. If directionality is desired, it is achieved by using multiple transducers, which limits the number of possible different directions to a set of discrete values, usually small in number. Our approach is different: using a high-fidelity, high-bandwidth 6-DOF haptic device, vibrations along any DOF, or combination of DOFs, are generated, enabling us to define an exact axis for the directional haptic feedback. The principal aim of the present study is to show that by making the vibration itself asymmetric, we can generate a sense of directionality along any axis, producing the haptic impression of being pushed toward or away from a region of space.

\section{ASYMMETRIC SINUSOIDAL VIBRATION}

The directional vibration is generated by an asymmetric sinusoidal function, composed of two half periods $\omega_{1}, \omega_{2}$ of different length, so that the rising part is steeper than the falling part or vice versa. By asymmetric we mean asymmetric over time, not with asymmetric amplitude, as seen in Figure 1 and Figure 2. An object vibrating asymmetrically has to move faster during the shorter half period of the signal and therefore output more power than during the longer half period. The idea is that this will be perceived as a directional clue to a subject when applied to a haptic device. The following equations define an asymmetric sinusoidal function:

$$
\begin{gathered}
x:[0 . .2 \pi[ \\
\Delta:[-1 . .1] \\
\omega_{1}=\pi+\frac{\pi}{2} \Delta
\end{gathered}
$$

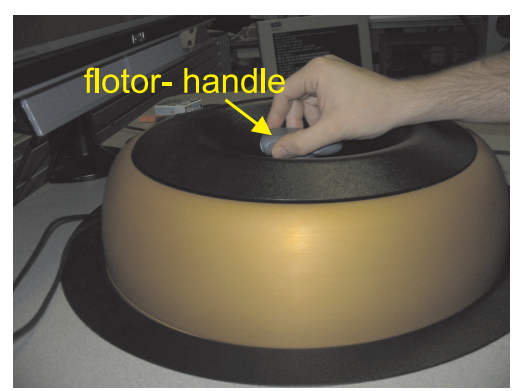

Figure 3: The Maglev Haptic device in front of a computer screen. The handle is rigidly attached to the flotor.

$$
\begin{gathered}
\omega_{2}=\pi-\frac{\pi}{2}(1-\Delta) \\
f(x)= \begin{cases}\text { if } x<\omega_{1}: & -\cos \left(x \frac{\pi}{\omega_{1}}\right) \\
\text { otherwise: } & -\cos \left(x \frac{\pi}{\omega_{2}}\right)\end{cases}
\end{gathered}
$$

$\omega_{1}$ is the half period of the sinusoidal function for the rising portion of the signal, $\omega_{2}$ the half period for the falling portion. $\Delta$ is the amount of asymmetry in the signal. For $\Delta=0, \omega_{1}=\omega_{2}$ and the resulting function is symmetric. The closer $|\Delta|$ gets to 1 , the more asymmetric the signal becomes. The difference between positive and negative $\Delta$ is the direction of vibration: a negative $\Delta$ is equivalent to rotating the desired axis of vibration by $\pi$.

\section{The Maglev haptic Interface}

The haptic interface used for this study is a newly developed desktop-mounted, 6-DOF magnetic levitation haptic device (MLHD, Figure 3, [7]). The device's only movable part, the flotor, is levitated in a strong magnetic field and tracked by optical position sensors.

The device exhibits extremely high fidelity due to the lack of friction and backlash. The high stiffness range (max. $40 \mathrm{~N} / \mathrm{mm}$ ) and a bandwidth of $130 \mathrm{~Hz}$ allow us to accurately generate the asymmetric vibrations for this study. Figure 4 shows an example of a slightly asymmetric function with frequency $6 \mathrm{~Hz}$ and $0.2 \mathrm{~mm}$ amplitude being tracked by the MLHD along the devices $\mathrm{x}$ axis.

\section{USER STUDY}

The goal for this user study (under CMU IRB HS08-316) was to determine subject's ability to identify the directions represented by asymmetric vibrations along different axes having different frequencies and amplitudes. Eleven subjects, all students, participated, 5 male and 6 female, between 19 and 26 years old. All subjects where untrained and not familiar with the use of the device or the test setup. All subjects where familiar with the general usage of a computer and a computer mouse. Before the test, a brief training session was performed: Subjects where presented with 3 random examples and told the corresponding direction.

Subjects sat in front of a computer screen. The MLHD Maglev haptic device was used to generate the vibrations as well as for user input. All parameters for the haptic device, like PID gains for position and orientation control, where constant over all tests.

\subsection{Test setup}

Figure 5 shows a screen capture of the test setup. In step 1 subjects felt an asymmetric vibration on the handle in the horizontal plane (xy). They were instructed to click a button on the handle after they thought they knew the direction of the vibration. Immediately a 


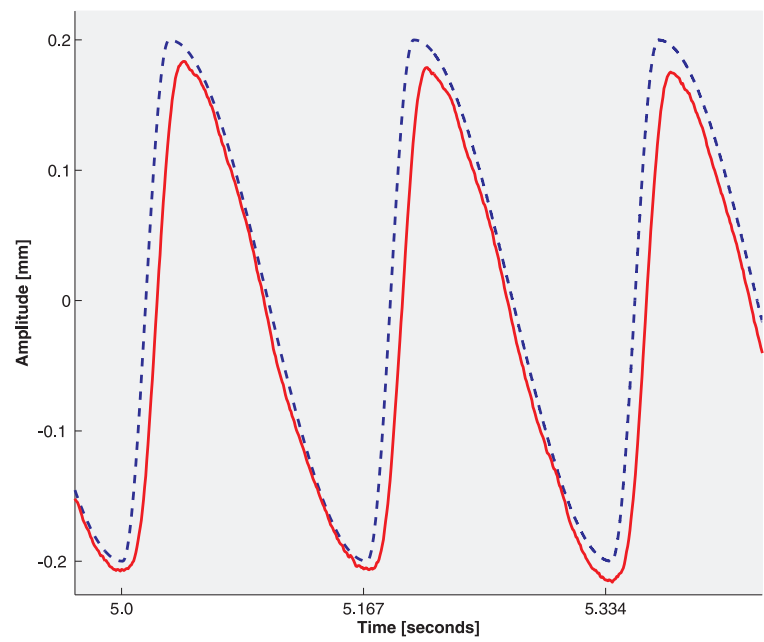

Figure 4: Tracking: $(6 \mathrm{~Hz}, 0.2 \mathrm{~mm})$, slightly asymmetric vibration. Blue is the desired function the device tries to track, red the actual position in $x$ of the flotor.

second screen with a dial appeared. While still feeling the vibration, subjects were to rotate the handle so as to adjust the dial to match its perceived direction. After clicking again, the next trial started. Each subject performed 192 trials.

\subsection{Stimulus directions}

Our focus was on 8 different directions in the xy-plane at $\frac{\pi}{4}$ intervals; however, to ensure that this did not become a classification task for the subjects, where they just choose between 8 discrete angles, only $75 \%$ of all trials (144 out of 192) where chosen from those 8 directions, the other $25 \%$ being chosen randomly from angles in between.

The following equations where used to compute the asymmetric sinusoidal vibration along an axis in the xy- plane of the haptic device, where $A$ is the amplitude of the signal and $\alpha$ the angle of the desired direction of vibration. asymsin() is an implementation of the function described in section 2 to generate the asymmetric vibration with the time $t$ in seconds, frequency $f$ in Hertz and amount of asymmetry $\Delta$.

$$
\begin{aligned}
& x=A \times \cos (\alpha) \times \operatorname{asymsin}(t, f, \Delta) \\
& y=A \times \sin (\alpha) \times \operatorname{asymsin}(t, f, \Delta)
\end{aligned}
$$

\subsection{Frequency-Amplitude pairs}

To compare performance at different frequencies and amplitudes, we selected 3 frequency- amplitude pairs for the trials. We were particularly interested in vibrations with very small amplitudes, in order to produce subtle feedback that would not disturb control, that is the average position and orientation of the handle over time. The frequency- amplitude pairs where chosen so that the output power of all 3 vibrations was the same:

- Pair A: $0.6 \mathrm{~mm}$ amplitude, frequency $2 \mathrm{~Hz}$

- Pair B: $0.4 \mathrm{~mm}$ amplitude, frequency $3 \mathrm{~Hz}$

- Pair C: $0.2 \mathrm{~mm}$ amplitude, frequency $6 \mathrm{~Hz}$

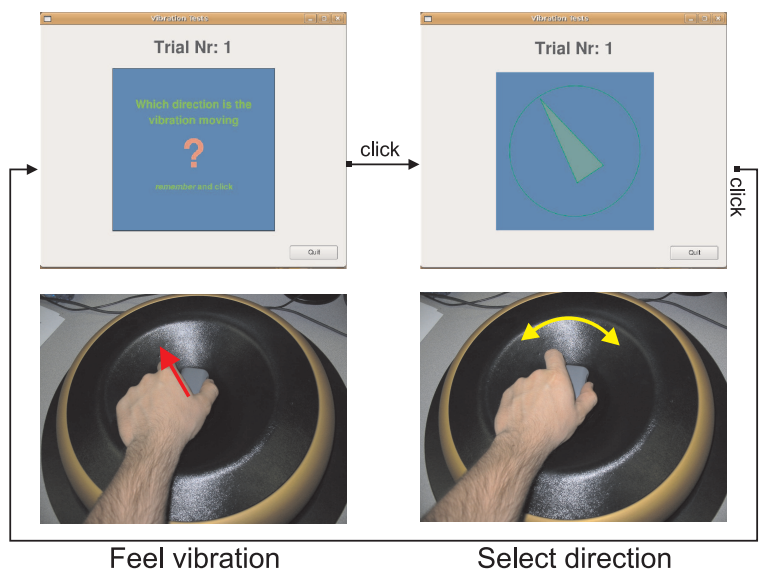

Figure 5: Test setup: subjects feel a vibration, then select its perceive direction by rotating the handle to adjust an on-screen dial.

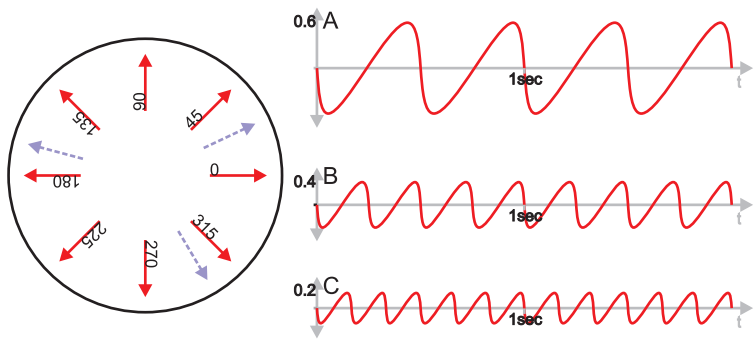

Figure 6: Trial parameters: The 8 main directions in which the test was designed to generate asymmetric vibrations (red arrows) and $25 \%$ random other directions (blue arrows). Right: the three frequency- amplitude pairs used in the trials, all with equal power. 


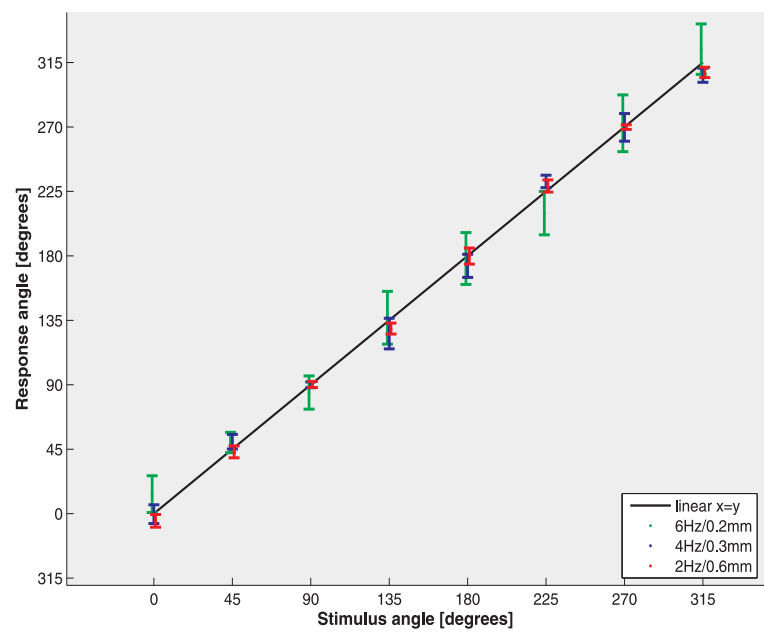

Figure 7: Regression plots: Actual direction of vibration $(x)$ vs. reported direction $(y)$ for all subjects. The error bars in show the means and standard error of the trials for the parameter sets $(2 \mathrm{~Hz}, 0.6 \mathrm{~mm})$ (red), (4 Hz, $0.3 \mathrm{~mm}$ ) (blue) and (6 Hz, $0.2 \mathrm{~mm}$ ) (green).

\subsection{Trials}

Each subject performed 6 repetitions per direction (8), per frequency- amplitude pair (3), for a total of $6 \times 8 \times 3=144$ trials, plus another 48 trials with random other directions, resulting in a total number of 192 trials. The order of trials was chosen at random by the computer. The trial information (direction, frequency and amplitude) was logged together with the angle indicated by the subject and how long it took the subject to respond. The asymmetry level of the vibrations was constant over all trials at 0.96 , constituting a strong asymmetry as described in section 2 .

\section{Results}

In a small number of trials (6) subjects reported the correct axis, but the wrong direction, meaning the guessed orientation was close to 180 degrees off. These trials were discarded from further data analysis, since they are not comparable to the small angle offsets otherwise observed. Figure 7 shows the mean response by subject with standard error bars for each condition. An ANOVA on stimulus angle $(8$ values $) \times$ amplitude- frequency combination $(3$ levels $)$ found strong effects of angle, $F(7,70)=126231, p<0.001$, reflecting a linear trend $\left(R^{2}\right.$ for each condition $\left.>0.99\right)$. The effect of amplitude- frequency combination was not significant but interacted with the angle, $F(14,140)=2.93, p<0.01$. This interaction reflects small differences among the conditions at some angles, but no reliable trend.

Responses were timed from the onset of the stimulus until the subject clicked a button to advance to the next screen and adjust the dial, as shown in Figure 5. An ANOVA on stimulus angle (8 values) $\times$ amplitude- frequency combination (3 levels) found effects of angle, $F(7,70)=7.52, p<0.001$, and condition, $F(2,20)=10.52, p<0.01$. The mean time tended to increase with angle (for the linear trend, $F(1,10)=10.10, p<0.05$ ), but time was also modulated by angle in a nonlinear fashion. The interaction did not reach significance.

A third measure was the within-subject standard deviation, computed over the six responses at each angle and amplitude/frequency.

An ANOVA on stimulus angle ( 8 values $) \times$ amplitude- frequency combination (3 levels) found effects of angle, $F(7,70)=$ $2.17, p<0.05$, and condition, $F(2,20)=31.89, p<0.001$. The effect of angle primarily reflects reduced variability when responses lay along the subject's sagittal axis. The effect of amplitude- fre-

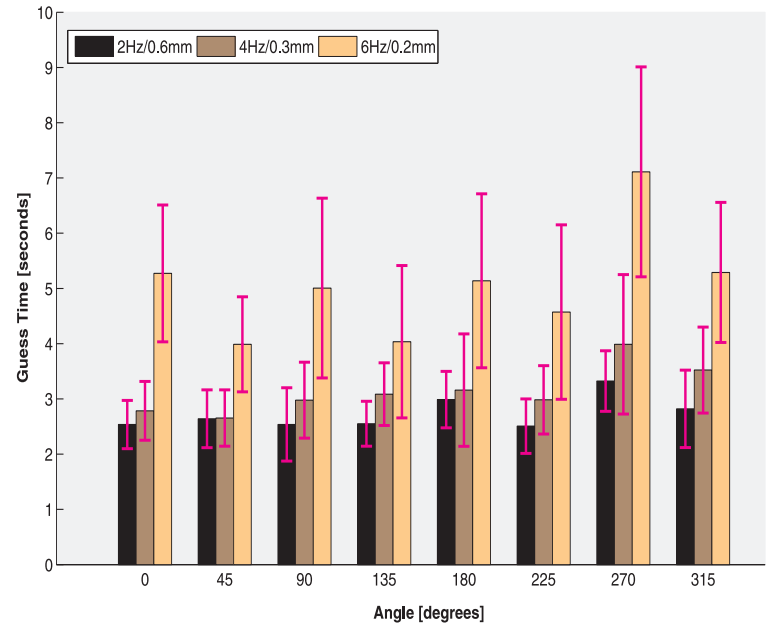

Figure 8: Response time: Average time in seconds for subjects to respond to a stimulus. The error bars (magenta) show the standard error of the mean.

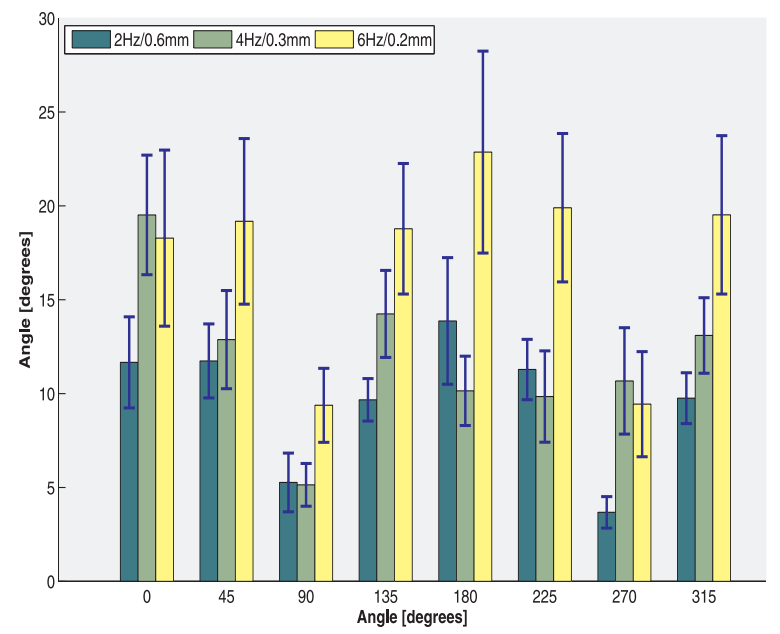

Figure 9: Mean S.D.: Shows how spread subject's responses where on average: Mean of the within-subject standard deviations for trials with a common angle and amplitude- frequency. The error bars (blue) show the standard error of the mean. 
quency was similar to that on time: The SD tended to increase with frequency, being particularly larger for the $(6 \mathrm{~Hz}, 0.2 \mathrm{~mm})$ condition. The interaction did not reach significance.

\section{Conclusion}

The analysis of the user study indicates that the system presented in this paper can produce accurate directional haptic cues using asymmetric vibrations. To make this useful, a device is required which can produce such vibrations. Subjects were highly accurate at reproducing the 8 major directions at $\frac{\pi}{4}$ intervals. It is possible that they were responding categorically to these angles, but this should have been discouraged by the large number of "dummy" trials presented at other angles. It is also worth noting that accuracy was not lower at the oblique angles, in contradiction to the well known Oblique Effect (described for example by Gentaz et al. in [8] and [9]).

Accuracy varied little with frequency/amplitude combination, but with higher frequency and lower amplitude, the subjects' performance became slower and more variable. There was a particularly sharp decline in performance - both speed and variability - with the amplitude/frequency combination of $(6 \mathrm{~Hz}, 0.2 \mathrm{~mm})$, where subjects were slower by $50 \%$ to $100 \%$.

Individual subject's mean ranged from 9 to 25 degrees, and the response time ranged from 1.9 to 8.0 seconds. Subjects who were slower were not particularly accurate, however; error actually correlated positively, though not strongly, with response time $(r=0.30)$. It would be useful to compare the performance variability from this study, using minimally trained subjects, with that of subjects who completed more extensive training, commensurate with daily use of this type of feedback. Presumably, experience would lead to faster, more stable performance.

\section{Discussion AND FUTURE WORK}

\subsection{Comparing system performance}

We are very interested in further exploring asymmetric vibrations for directional haptic feedback and comparing user performance with other haptic methods such as feedback from continuous forces.

While the present work applies asymmetric vibrations to a user's hand in the xy-plane, it should be straightforward to apply the same technique in 3-DOF or even in all 6-DOF, to guide subjects through higher-dimensional work spaces.

Vibrations offer one more dimension for feedback in comparison to continuous forces: time. As was mentioned above, feedback can be intensified by both higher amplitudes and larger frequencies, and it would be valuable to systematically explore the amplitudefrequency parameter space.

In this study, we used fairly low frequencies for the vibrations to make sure the device is tracking the commanded motions exactly. It would be very interesting to see how subjects react to higher frequency asymmetric vibrations (with smaller amplitudes).

\subsection{Applications}

We are also interested in applying haptic feedback via asymmetric vibrations to real-world tasks. We believe that asymmetric vibrations with fairly small amplitudes are well suited to be superimposed with other haptic feedback, for example with continuous forces, and with control commands when the haptic device is used both as an input and output device at the same time.

Possible applications could be controlling manipulators or mobile robots, where asymmetric vibrations could indicate distance (amplitude and/or frequency) to an obstacle and its location by hinting the direction in which the obstacle is relative to the robot. Alternatively, suggested actions could be hinted to the operator like turn in place, move faster/slower, and variable amplitude and frequency could hint amount and urgency.

\section{ACKNOWLEDGEMENTS}

This work was supported in part by NSF grant IIS-0413085.

\section{REFERENCES}

[1] Robert W. Lindeman, John L. Sibert, Erick Mendez-Mendez, Sachin Patil, and Daniel Phifer. Effectiveness of directional vibrotactile cuing on a building-clearing task. In Proceedings of ACM CHI, volume 1, April 2005.

[2] Cristy Ho, Hong Z. Tan, and Charles Spence. Using spatial vibrotactile cues to direct visual attention in driving scenes. In Transportation Research Part F: Traffic Psychology and Behavior, volume 8, page 397412, April 2005.

[3] A.M. Okamura, M.R. Cutkosky, and J.T. Dennerlein. Reality-based models for vibration feedback in virtual environments. IEEE/ASME Transactions on Mechatronics, 6:245-252, 2008.

[4] A.M. Okamura, J.T. Dennerlein, and R.D. Howe. Vibration feedback models for virtual environments. In Robotics and Automation, 1998. Proceedings., volume 1, page 674679, May 1998.

[5] Amemiya T., Ando H., and Maeda T. Virtual force display: Direction guidance using asymmetric acceleration via periodic translational motion. In Proceedings of Euro Haptics 2005, volume 1, May 2005.

[6] Tomohiro Amemiya and Taro Maeda. Asymmetric oscillation distorts the perceived heaviness of handheld objects. IEEE Transactions on Haptics, 1:9-18, 2008.

[7] P. J. Berkelman, Z. J. Butler, and R. L. Hollis. Design of a hemispherical magnetic levitation haptic interface device. In Proc. ASME Symposium on Haptic Interfaces, volume DSC-58, pages 483-488, Atlanta, November 17-22 1996.

[8] Edouard Gentaz and Yvette Hatwell. The haptic 'oblique effect' in children's and adults' perception of orientation. Perception, 24:631646, 1995.

[9] LUYAT Marion, MORONI Christine, and GENTAZ Edouard. The role of contextual cues in the haptic perception of orientations and the oblique effect. Psychonomic bulletin and review, 12:760-766, 2005. 